\title{
La construcción de la categoría ecorregión en los docentes en formación. "Una propuesta de aprehensión y apropiación de realidades"
}

\author{
María Rocío Pérez Mesa
}

Artículo recibido: 27-05-2005 y aprobado: 26-04-2006

The construction of the category "ecorregion"

in preservice teachers. "A proposal of apprehension and appropriation of realities"

Resumen: Esta investigación tiene como propósito hacer una conceptualización de la categoría ecorregión, como posibilidad de construcción de conocimiento y punto de partida para la comprensión de realidades ambientales en los docentes en formación.

Palabras clave: Construcción de conocimiento, ecorregión, categoría, aprehensión, ambiente, territorio, región, educación ambiental

Abstract: The main aim of this research is to make a conceptualuization of "ecorregion" category as a possibility of knowledge construction and as our starting point for the understanding of environmental realities with preservice teachers.

Key words: Kmowledge construction, ecorregion, category, apprehension, environment, territory region, enviromental education.

Profesora, Departamento de Biología Universidad Pedagógica Nacional. Magistra en Educación con énfasis en Educación Ambiental. rociopm28@yahoo.es 


\section{Introducción}

El presente trabajo recoge algunos principios de la educación ambiental orientados hacia la comprensión de las interrelaciones que se dan en los sistemas ambientales, de acuerdo con un tiempo y un espacio determinados (contexto). Es aquí donde los sistemas vivos y los sistemas sociales, en tanto sistemas complejos (Morin, 1996), presentan unas dinámicas e interacciones que en su seno dan lugar a transformaciones, traducidas en orden, desorden y organización. En este sentido, se pretende propiciar un espacio de discusión en donde se privilegie el análisis de las visiones de mundo y la construcción de conocimiento de los docentes en formación, que favorezcan la toma de decisiones y el establecimiento de un compromiso responsable con la vida y la sostenibilidad.

El trabajo desde la ecorregión se convierte en oportunidad para crear situaciones de diálogo, en diferentes espacios relacionales, tomando como punto de partida el escenario que brinda la Universidad Pedagógica Nacional. Este proceso busca fortalecer en los estudiantes la argumentación frente a las nuevas propuestas para un manejo ambiental adecuado y un desarrollo humano integral. De esta forma, la construcción de conocimiento, la aprehensión y apropiación de sus realidades desde los contextos social, natural y cultural, se constituyen en un referente para la reconstrucción de esos conocimientos a través de su quehacer docente, fundamentados en la responsabilidad y el compromiso consigo mismo, con el colectivo y con el ambiente en general.

\section{La necesidad de una conceptualización de ecorregión}

En la época actual, es aceptado reconocer que somos testigos y protagonistas de un período de transformaciones y cambios analizados desde diferentes dimensiones del actuar humano. Hacemos parte del hecho que se investiga, y como tal manifestamos una relación inseparable con las propiedades que emergen en cada nivel de interacción con el colectivo, el ambiente y nosotros mismos. Nuestra capacidad de repensar el mundo nos permite identificar las problemáticas ambientales que surgen de dichas interacciones, con lo que se toma conciencia desde la colectividad sobre los conocimientos, los valores, las competencias, la experiencia y la voluntad, y actuar individual y socialmente, con el fin de reconstruir los procesos de comunicación involucrados en la consolidación de una cultura ambiental.

Es importante para el abordaje de las problemáticas ambientales tener en cuenta diversas perspectivas que abarcan lo biofísico, lo económico, lo social, lo político, lo formativo, lo cultural, lo geográfico y lo histórico, razón por la cual debe complejizarse la mirada de la ecorregión como categoría que trasciende el significado de territorio y región, para comprender las transformaciones ecosistémicas y los impactos ocasionados por la actividad humana.

Así, desarrollar alternativas de conservación y restauración de áreas prioritarias en el marco de un desarrollo sostenible local, amparadas en un saber reconstructivo consolidado en la pedagogía, permite crear un espacio de discusión académica en la Universidad, 
que tenga en cuenta la posibilidad de construcción de conocimientos, el fortalecimiento de procesos cognitivos y la profundización teórica y metodológica en relación con la categoría de ecorregión. Con esto se fortalece la argumentación en torno a nuevas propuestas para un manejo adecuado del ambiente a partir de la construcción de criterios de actuación frente a las complejas problemáticas ambientales que requieren un tratamiento crítico y reflexivo desde la academia, para lo cual los docentes en formación se convierten en dinamizadores de la cultura al fomentar la ética, la responsabilidad, el conocimiento, los valores y la toma de decisiones a las generaciones presentes y futuras.

En este sentido, la propuesta de ecorregión planteada por el Ministerio de Ambiente, Vivienda y Desarrollo Territorial se limita a una definición político-administrativa como se expresa a continuación:

Unidad territorial definida con base en tres criterios principales derivados de los objetivos de la política ambiental y de los instrumentos para su implementación:

- Delimitación territorial, de manera que relacione la oferta ambiental presente en unidades ecológicas prioritarias para la retención y regulación del agua, con la demanda generada en áreas urbanas o rurales.

- La condición, que este territorio sea compartido por varias Corporaciones Autónomas Regionales o entes territoriales, lo que las convierte en elementos articuladores y dinamizadores del trabajo colectivo en torno al ambiente, $y$
- Su carácter estratégico, definido por la posibilidad de articular su oferta natural a la solución de los conflictos actuales relacionados en forma inmediata con los principales procesos y objetivos económicos y sociales contemplados en el Plan Nacional de Desarrollo, Cambio para Construir la Paz. (Proyecto Colectivo Ambiental, 2000).

Por tanto, uno de los propósitos de la presente investigación consiste en elaborar una conceptualización de ecorregión como categoría, definida por Zubiría (1997) como "un sistema de conceptos relacionales", en dos instancias: la primera, elaborada por los docentes en formación, y la segunda, producto de la reflexión, la retroalimentación y la discusión propias de esta investigación. Así, la propuesta avanza hacia otros dos propósitos: la invitación a una discusión de la categoría por parte de la comunidad académica, y la consideración de dicha categoría como un elemento fundamental dentro de las discusiones acerca de educación y gestión ambiental.

\section{Aportes desde la educación ambiental}

Dentro de la discusión en torno a la construcción de la categoría ecorregión, se hace perentorio iniciar una serie de reflexiones acerca de la importancia de desarrollar una mirada amplia, sistémica y compleja, que permita el fortalecimiento de los procesos cognitivos de los estudiantes. Esto a partir de la implementación de estrategias pedagógicas que pongan en diálogo esos saberes y esas formas de construir el conocimiento mediante un aprendizaje significativo (Ausubel, 1978), en un 
escenario académico que trascienda las aulas y establezca otros espacios de trabajo, convirtiéndose en una propuesta metodológica para la gestión ambiental, en la que se invite al encuentro del sujeto consigo mismo, con los otros y con el entorno, como un espacio democrático y plural. Este proceso hace posible la legitimación del sujeto, por cuanto se constituye en nodo de ese espacio relacional, en el que se dinamizan procesos de retroalimentación que permiten, a su vez, contextualizar los referentes sobre los cuales se fundamenta la investigación, al hallar sentidos y construir significados para la comprensión de realidades ambientales.

Se piensa entonces en la importancia y el papel preponderante que desempeña la educación ambiental como una manera de ver el mundo (Novo, 2003), en la que se construyen conocimientos y se revalidan las relaciones entre los individuos y el entorno, en donde los principios éticos permiten dimensionar las interrelaciones entre el sistema natural y cultural, su complejidad, sus conflictos e interdependencias, hecho que fortalece el asumir una conciencia ambiental necesaria para contribuir al cambio en las relaciones de los sujetos con el ambiente (Torres, 1996).

Lo anterior, lleva a formular desde la educación -y en particular desde la educación ambiental-, un trabajo amplio y comprometido a partir de la reflexión crítica, orientado a la formación de diversos actores, tanto en lo referente a la lectura de contextos ambientales, como en lo referente a la comprensión de la categoría ecorregión.

\section{La construcción de la categoría ecorregión}

Durante el proceso de construcción de la categoría ecorregión se tuvieron en cuenta los siguientes aspectos:

- La dimensión integral del ambiente y la dimensión del desarrollo sostenible, requerido en la preservación de la diversidad biológica, cultural y territorial del contexto de estudio.

- La generación de situaciones tendientes a propiciar la reflexión en torno a la búsqueda de soluciones a los problemas ambientales.

- La importancia de resaltar la política ambiental del Proyecto Colectivo Ambiental (PCA), que propende a la restauración y la conservación de áreas prioritarias en ecorregiones estratégicas a través del desarrollo sostenible a nivel sectorial y regional.

- La pertinencia de conceptualizar el ambiente (Talero y Umaña, 1998), el territorio y la región (Rodríguez, 2000), para comprender las interrelaciones de éstos en un espacio y tiempo determinado, así como el estudio de los sistemas agua, bosque $\mathrm{y}$ biodiversidad, que requieren un manejo adecuado, orientado hacia la sostenibilidad y conservación del medio. Vale la pena resaltar el tratamiento de aspectos socioculturales relacionados con la calidad de vida, a través de la apropiación de conceptos, la interpretación de interrelaciones, la identificación de problemáticas y el planteamiento de posibles soluciones para el manejo adecuado del sistema ambiental, pensado a partir de la ecorregión. 


\section{Metodología}

De acuerdo con los propósitos del presente trabajo, se propone como enfoque la investigación acción (IA), que permite ampliar la mirada frente a las interacciones y desarrollos que se entretejen con el grupo de estudiantes de cuarto semestre de la Licenciatura en Ciencias Sociales, frente al estudio y la construcción de la categoría ecorregión.

Según Arnal et al. (1992), la investigación-acción se constituye en un medio para desarrollar la capacidad de resolver problemas por parte del profesor, y en una metodología para elaborar currículo y formar al profesorado.

De esta forma, la investigación-acción está entroncada con los criterios y las premisas de la lógica de la investigación interpretativa. Cada proceso de investigación-acción interpreta lo que ha ocurrido en la acción en relación con el contexto; pero lo interpreta desde el punto de vista de los que actúan e interactúan en la situación problema. El resultado de cada ciclo de la espiral investigación-acción presenta el desarrollo general del proceso, exponiendo las ideas y los principios clave de la acción, los problemas encontrados, las técnicas utilizadas para recoger la información, lo cual sirve de base para la reflexión de la acción educativa.

En este sentido, la investigación-acción requiere el trabajo en colaboración de todos los implicados en el proceso de investigación. Su fuerza epistemológicointerpretativa se amplifica hacia una dimensión abiertamente ético-democrática, dado que quienes participan en el proceso se constituyen en protagonistas y constructores del conocimiento y de su realidad. Así mismo, su naturaleza flexible hace de la retroalimentación un elemento propicio para la reflexión y la creación de consensos abiertos al diálogo, además de generar un pensamiento crítico y resolutivo. Por tanto, este trabajo parte de los principios metodológicos de la IA, que fundamentan la investigación acerca de la construcción de la categoría ecorregión en los docentes en formación.

\section{Muestra}

La muestra se encuentra constituida por un grupo de 30 estudiantes, de cuarto semestre de la Licenciatura en Ciencias Sociales de la Universidad Pedagógica Nacional, quienes en semestres anteriores tuvieron cierto tipo de desarrollo relacionados con el núcleo temático: “Tiempo, espacio y sociedad". Dentro de este núcleo temático se abordan componentes como:

- Sistemas espaciales de Colombia, que tiene por objeto formar un pensamiento sistémico orientado a la comprensión y al análisis estructural de los diferentes sistemas espaciales del país.

Aquí se pone de manifiesto el estudio de los sistemas abiertos, por lo que se complejiza la dinámica de los sistemas geográficos, y con ello la comprensión y el dominio de la organización territorial, el establecimiento de interacciones entre la oferta biofísica y la oferta social (a través del tiempo y el espacio) y el conocimiento de la realidad multidimensional para organizar territorios espaciales.

- Sociedad y medio ambiente, en el cual se intenta comprender el concepto de cultura desde lo ambiental, así como los impactos que ocasionan las actividades humanas en el medio na- 
tural. Además se contempla el análisis de conflictos y oportunidades para lograr un desarrollo sostenible local.

Como vemos, es importante destacar una progresión a partir de la interacción entre los conocimientos que el estudiante posee y aquellos que le brinda la escuela; así mismo, construir un concepto puede resultar más fácil cuando existen motivaciones internas y profesionales, así como cuando se reconoce la importancia del mismo. En este sentido, la construcción de la categoría ecorregión se hace factible al ser estructurante y pertinente en la formación de dichos estudiantes.

\section{Fases y estrategias pedagógicas Diagnóstico}

Dentro de esta fase, resulta indispensable reconocer la trayectoria académica de los estudiantes en relación con el pénsum propio de la carrera, de manera que permita una mejor contextualización del trabajo, para partir de las ideas alternativas que presenta el grupo acerca del ambiente, territorio, región y ecorregión, empleando como estrategia la aplicación de un taller de indagación.

\section{Proceso de construcción de la categoría}

En esta fase se desarrollan estrategias pedagógicas como el taller y la salida de campo, para que se propicie una mayor interacción con el grupo de docentes en formación, dando lugar al establecimiento de espacios de trabajo pedagógico que favorecen los procesos de estructuración permanente a nivel individual y colectivo.

En este sentido, el taller se constituye en un espacio de diálogo en el que intervienen todos los participantes y favorece el desarrollo de las actividades, la solución a preguntas y problemas, el planteamiento de interrogantes y la motivación por participar al ser éste un tema de interés para ser abordado por el grupo.

Así mismo, la salida de campo se convierte en otro espacio para la construcción de la categoría ecorregión, dado que el trabajo ambiental no podría concebirse sin estudios sobre el terreno (Umaña, 2004), pues conviene desarrollar la experiencia directa en el entorno, permitiendo que el docente en formación haga una lectura del sistema, establezca relaciones, interprete las realidades que se circunscriben en dicho espacio, identifique las problemáticas que emergen y reflexione sobre posibles alternativas de solución desde el ejercicio docente.

Las temáticas por seguir en los talleres y las salidas de campo comprenden dos ejes centrales: el sistema agua, bosques y biodiversidad y su relación con la calidad de vida y la sostenibilidad a nivel local y regional.

\section{Conceptualización en torno a la categoría ecorregión}

Para esta última fase es importante destacar la concreción de la conceptualización que los docentes en formación hacen de la categoría ecorregión, a partir de todo el proceso desarrollado a través de talleres y salidas de campo, los cuales han permitido en dichos sujetos enriquecer sus miradas al facilitar las interacciones sujeto-sujeto, sujetocontexto, facilita que los aprendizajes obtenidos en ella se conviertan en constructivos y reelaboren la estructura 
semántica, de modo tal que la modifican y la preparan para un nuevo proceso de aprendizaje, (Novo, 1991).

En esta fase, los estudiantes se organizan por parejas o grupos de tres personas, y en una hoja de papel dan respuesta a la pregunta: ¿Qué comprenden por ecorregión?

Posteriormente se realiza la puesta en común para socializar y reflexionar acerca de la construcción a la que ha podido llegar el grupo de estudiantes, y comprender la importancia de la ecorregión en su proceso de formación y proyección profesional.

\section{Resultados y análisis}

Los resultados obtenidos de las fases investigativas se presentan y analizan a continuación, teniendo en cuenta las elaboraciones de los protagonistas, quienes participaron y dinamizaron el trabajo a partir del reconocimiento de sus ideas alternativas, los procesos reflexivos, el pensamiento crítico y resolutivo frente a las problemáticas ambientales del contexto de estudio, y la toma de decisiones que se concretan en la conceptualización de la ecorregión.

\section{Fase 1. Indagación de las ideas alternativas}

Es importante mencionar que esta fase trasciende la indagación de las concepciones de los estudiantes, dado que a su vez se constituyó en un escenario propicio para la discusión, la reflexión y la problematización acerca de las elaboraciones iniciales y la estructuración hacia unas conceptualizaciones más complejas y significativas gracias a una permanente retroalimentación como elemento fundamental en la investigación-acción.

\section{Ambiente}

- El 50\% de los estudiantes representa el ambiente como un espacio natural, constituido por factores bióticos y abióticos en donde no se incluye el hombre. En este sentido, presentan una idea de naturaleza pura para respetar y preservar en la medida en que el hombre toma distancia de la misma. Estas formas de pensar el ambiente se relacionan con las ideas que circulan en la cultura dentro de la cual han interactuado los estudiantes teniendo un peso considerable la perspectiva ecológica -y en este caso ecosistémica- que fragmenta el ambiente, y en la que el hombre y la cultura se desdibujan.

- El 33\% concibe el ambiente como un recurso para aprovechar y satisfacer las necesidades del hombre, y también le provee las condiciones para vivir y coexistir con otras formas de vida.

- El 17\% considera el ambiente como un espacio para indagar y comprender sus dinámicas, sus interrelaciones y la diversidad que puede presentar. De acuerdo con lo expresado por los estudiantes, es importante destacar que hacen explícito el establecimiento de relaciones entre el hombre y lo biofísico, situación que puede entenderse por las estructuraciones que han logrado establecer en su formación académica.

\section{Territorio}

- El 47 \% ve el territorio como un espacio geográfico, y lo representa mediante el mapa de Colombia.

- El 37\% hace explícitas las ideas de pensar el territorio no sólo como un espacio geográfico, sino que a su vez 
vincula al hombre como individuo y colectivo que apropia y valora dicho territorio, y construye una identificación con el mismo.

- El 16\% presenta al territorio como herramienta para ejercer el control de carácter político, que posibilita la administración y regulación del mismo. En un alto porcentaje las ideas expresadas por los docentes en formación muestran que se reduce a la delimitación de un espacio en donde se ejerce un poder, dejando de lado las construcciones sociales y culturales que implica la construcción del mismo.

\section{Región}

- El 70\% de los estudiantes concibe la región como un área delimitada y con relativa homogeneidad biofísica.

- El 27\% asume la región como un sistema dinámico, transformado por la interacción de estructuras sociales, territoriales y culturales, lo cual llama la atención porque en la conceptualización que hacen de la región por escrito expresan en un $51 \%$ estas interrelaciones, lo cual muestra una diferencia considerable con las ilustraciones que hacen de la misma.

- Se mantiene en un porcentaje del 3,33\% la identidad como aspecto por considerar al momento de representar la región. El sujeto cognoscente se reconoce como parte de ella y expresa valores de apropiación, lo cual es compatible con lo expresado en el escrito.

De acuerdo con las respuestas dadas por los estudiantes acerca de lo que es para ellos la región, se observa cierta claridad en el manejo del concepto de región, que permite evidenciar la in- fluencia de su formación en el proceso de aprendizaje y la experiencia escolar adquirida en los semestres anteriores, reflejo de su propio proceso de construcción.

\section{Ecorregión}

Al responder la pregunta: ¿Qué entiende por ecorregión?, las respuestas dadas por los estudiantes se organizaron en cinco categorías que muestran las tendencias de dicho concepto, a saber:

\section{La ecorregión como espacio homogéneo:}

\section{2 estudiantes}

- Prima el referente de homogeneidad expresado en un 40\%, determinado por la oferta biofísica, lo cual permite entrever que los estudiantes conjugan elementos de los conceptos de ecosistema y región para estructurar y dar sentido a la pregunta acerca de qué concepción tienen de ecorregión; además realizan una distinción entre los elementos y componentes que la constituyen.

De acuerdo con las ideas alternativas que presentan los estudiantes y con los desarrollos alcanzados en el transcurso de su carrera, plantean la ecorregión como un espacio delimitado, dinámico y homogéneo principalmente. Dicha homogeneidad está dada por las características biofísicas, y en segundo lugar contemplan lo cultural. Ej.: "Es un espacio geográfico homogéneo determinado por la diversidad en fauna y flora".

La ecorregión como sistema de relaciones: 4 estudiantes

- Para el 13\%, la ecorregión es pensada en términos de relación entre lo biótico y lo abiótico; haciendo poco énfasis en el reconocimiento del 
hombre como parte del componente biótico; por tanto, no se dimensiona la importancia que tiene éste en los procesos de transformación y establecimiento de nuevas dinámicas en el ambiente.

- Llama la atención que los estudiantes muestran una idea de sistema al ir más allá de listar unos elementos y agruparlos en un conjunto, para empezar a hablar de componentes que interactúan, lo cual supone una afectación mutua y la generación de nuevas dinámicas y posibles transformaciones en éste. Ej.: "Es un término que agrupa la interacción entre la parte física-biológica y antrópica en un espacio específico".

La ecorregión como sistema natural: 8 estudiantes

- El 27\% de los estudiantes consideran como base de la ecorregión el sistema natural desde sus componentes bióticos y abióticos sobre el cual se encuentra estructurada la vida y los componentes necesarios para su desarrollo, dando lugar a una amplia diversidad, lo que permite caracterizar y diferenciar un espacio. Ej.: "Es un conjunto de elementos y características propias de un sistema natural".

La ecorregión como espacio de la acción humana y del desarrollo: 5 estudiantes

- El 17\% de los estudiantes involucra al hombre dentro de la ecorregión, en términos de su actuar, a partir de las construcciones de la humanidad manifestadas en la cultura, como un mecanismo de desarrollo y de transformación sobre el ambiente, lo cual puede hacerse explícito en la generación de impactos positivos o negativos en el mismo. Ej.: "Es una unidad dinámica en la que convergen elementos bióticos, abióticos y culturales bajo la influencia de la transformación humana".

La ecorregión como asociación de conceptos: 1 estudiante

- El 3,3\% desglosa la preconcepción de ecorregión en dos partes: el prefijo eco lo relaciona con el concepto de ambiente, y el sufijo con el concepto de región, involucrando a su vez el concepto de territorio; tal situación permite entrever tímidamente una relación de estos conceptos con lo que puede ser la ecorregión. Ej.: "Concepto asociado al ambiente, territorio y región".

\section{Fase 2. proceso de construcción de la categoría ecorregión}

El trabajo desarrollado durante esta fase, a través de los talleres y las salidas de campo relativos al estudio y a la comprensión de las dinámicas y relaciones del sistema agua, bosques y biodiversidad, así como de la calidad de vida y la sostenibilidad del contexto de estudio, contempló una serie de actividades interrelacionadas, correspondientes a la producción de textos escritos, elaboración de mapas conceptuales, ubicación espacial, dramatizaciones, resolución de problemas, diligenciamiento de matrices acerca del sistema ambiental objeto de estudio, entrevistas a los habitantes de la región, reconocimiento sobre el terreno de las problemáticas asociadas a los ejes centrales de estudio, y su interpretación y retroalimentación permanentes. 
De esta manera, y de acuerdo con los resultados obtenidos, se evidenció la progresión de las concepciones hacia formas más complejas de comprensión por parte de los sujetos en torno a la categoría ecorregión.

Se puede destacar en esta fase que la implementación de las salidas de campo permite a los estudiantes confrontar las concepciones y los referentes teóricos con una realidad ambiental contextual, además de tener la posibilidad de establecer otros niveles de complejización que hacen posible la identificación de emergencias en dicho sistema, así como procesos de reflexión que involucran la negociación de significados (Moreira, 2003) a partir del diálogo, de modo que esa interacción sujeto-entorno favorece los aprendizajes obtenidos en ella, convirtiéndose en constructivos; es decir, que de forma recurrente vienen a reelaborar la propia estructura semántica, de modo que permiten reorganizar la trama cognitiva del docente en formación y con ello la transformación en sus maneras de interpretar el mundo y de ubicarse en el mismo.

\section{Fase 3. construcción de la categoría ecorregión por los docentes en formación} A continuación se transcriben dos de las diez conceptualizaciones de ecorregión elaboradas por los estudiantes divididos en subgrupos de tres integrantes, dado que la presentación en su totalidad resultaría bastante extensa. De esta forma, las dos conceptualizaciones seleccionadas aleatoriamente permiten ilustrar el proceso desarrollado.

Espacio homogéneo con características biofísicas y culturales en el cual se construyen relaciones dinámicas y complejas en el que se sitúa el hombre como ente transformador del espacio, y creador de propuestas de intervención orientadas hacia un manejo sostenible de los recursos e implementación de producciones limpias, en relación con sus características ambientales contextuales y la satisfacción adecuada de las necesidades humanas, para mejorar la calidad de vida de la población.

Categoría de ecorregión $\mathrm{N}^{\circ} 2$

La ecorregión corresponde a un espacio diferenciado con características biofísicas similares, en donde se construyen relaciones sociales, dinámicas mutables y particulares teniendo como propósito comprender mejor las necesidades de los habitantes para mejorar la calidad de vida, impulsar los procesos productivos y la creación de mercados verdes que hagan compatible el desarrollo de la sociedad con el ambiente.

\section{Análisis acerca de la construcción de la categoría ecorregión}

De acuerdo con la progresión de las ideas de los estudiantes relativa a la construcción de la categoría ecorregión, es menester establecer aquellos razonamientos que surgen de la participación democrática de los sujetos en determinados núcleos de consensos, espacios en los que se explicita un cambio en la interpretación del mundo y, por tanto, una motivación por el reconocimiento de la urdimbre de significados que se entretejen ante la multiplicidad de marcos explicativos con los que los sujetos intentan comprender situaciones, de por sí, complejas.

Para construir la categoría ecorregión, los estudiantes parten del reconocimiento de las relaciones entre los conceptos de territorio, ambiente 
y región, con base en la delimitación geográfica, la homogeneidad biofísica y las características culturales comunes. $\mathrm{Al}$ expresar en éste la construcción de relaciones dinámicas y complejas, se evidencia la idea de cambio y tiempo que se complejiza de acuerdo con esa red de relaciones que se establecen entre lo social y lo biofísico, lo cual propicia diversas emergencias:

- El hombre se constituye en el actor principal al reconocer su capacidad transformadora mediada por su racionalidad y creación manifestadas en las prácticas culturales que ejerce en un contexto determinado, expresadas en este caso como propuestas de intervención. Dichas propuestas tienen como connotación el hecho de intervenir apropiadamente en el medio biofísico, a partir de la lectura adecuada del ambiente contextual, para hacer compatibles las formas de aprovechamiento de los recursos con la base ecosistémica, orientadas hacia el manejo sostenible.

- Los estudiantes incorporan a la conceptualización de ecorregión otro aspecto que resulta fundamental: la satisfacción de las necesidades para mejorar la calidad de vida, lo cual permite ver que al hablar de este tema los estudiantes no lo reducen a las actividades económicas sino que lo articulan a la satisfacción de las necesidades humanas, sintetizando en ello lo propuesto por Max Neef (2000), para pensar en un desarrollo a escala humana, y de esta forma dimensionar lo social en la ecorregión.
En este sentido, se manifiesta a partir de lo escrito la actividad constructiva que hacen los estudiantes, producto de un proceso en el que han sido partícipes mediante acciones vivenciales a través de cuestionamientos, búsquedas e indagaciones que les ha permitido establecer una serie de relaciones, expresadas en una construcción compleja, lo cual refleja los desarrollos alcanzados en relación con las concepciones que presentaban al iniciar el trabajo. Se observa que los aspectos abordados durante la segunda fase correspondiente al proceso de construcción de la categoría ecorregión resultaron ser significativos, puesto que les posibilitaron no sólo explicar situaciones con sus propias palabras y resolver problemas en un contexto particular, sino que lograron abstraer y conceptualizar la ecorregión.

\section{Categoría ecorregión № 2}

La ecorregión se asume como una construcción del hombre dentro de un marco conceptual político teniendo en cuenta como elementos la delimitación y diferenciación de espacios y la confluencia de las relaciones sociales, lo cual, hace posible ver la sociedad más allá de un conjunto de personas, para aterrizar la mirada sobre las relaciones que se tejen entre los individuos y grupos, pensados en términos de dinámicas mutables o quizás posibles emergencias.

Se piensa la ecorregión como una estrategia cuyo propósito está centrado en lo humano y el desarrollo, puesto que señala la importancia de entender las necesidades reales de los habitantes para que mejoren su calidad de vida, aunque no es claro quién o quiénes asumirían dicho compromiso (gobierno, 
grupos sociales, individuos), si es un proceso de participación y responsabilidad conjunta o es exclusividad del Estado atender a estas necesidades.

Por otro lado, se hace especial mención a la necesidad de generar un desarrollo compatible de la sociedad con el ambiente, apoyado en el impulso de los proyectos sostenibles y la creación de mercados verdes, lo cual permite ver en éstos las posibles alternativas al desarrollo, situación que no se puede desligar de las sociedades y de las formas de aprovechamiento de los recursos naturales.

De acuerdo con el desarrollo que los estudiantes hacen del concepto de ecorregión y lo expresado en los talleres se muestra que no sólo se han limitado a adquirir una información, sino a construir una forma de pensar la ecorregión al poner en juego un sistema de relaciones de orden político, biofísico, económico y social contextualizados en un espacio.

Cabe destacar que dicho concepto es estructurado dado su interés profesional y la afinidad con la carrera de licenciatura que se encuentran adelantando. Si bien se determina un escenario de trabajo, Ubaté, éste se convierte en un espacio de conocimiento desde la exploración del entorno que permite reconocer los diversos elementos que confluyen y emergen, así como la distinción de problemáticas en esa compleja trama que da cuenta de la ecorregión.

\section{Conclusiones}

- Uno de los aspectos relevantes en la presente investigación corresponde a la identificación de las ideas alternativas de los estudiantes frente al ambiente, el territorio, la región y la ecorregión. Éstos se constituyen en la base del trabajo, puesto que permiten, a partir de la indagación inicial, aproximarse a esas maneras de interpretar el mundo, al ser éstos los marcos de referencia que, en otras palabras, corresponden a la red de conocimientos, afectos y valores previamente establecidos por el estudiante. Esta trama influye de manera notoria en sus expectativas y actuaciones a la hora de aprender algún concepto, en sus bloqueos ante determinada información y, en definitiva, en poder relacionar de una manera no arbitraria aquello que aprende con aquello que ya sabe.

Es así que prevalece en los diferentes momentos de la fase de diagnóstico en la mayoría del grupo de docentes en formación una manera de concebir el ambiente desde la perspectiva ecológica -y en este caso ecosistémica-, que fragmenta el ambiente, y en la cual se desdibujan las relaciones con el hombre y la cultura. Esta situación muestra la tendencia que tienen los estudiantes a asimilar los conocimientos de manera acrítica, desconociendo su significado y sentido, siendo consecuencia de ello la incomprensión.

Por otra parte, las redes que los estudiantes presentan para interpretar la realidad son idiosincráticas, y desde ellas se debe hacer la indagación, puesto que la construcción del conocimiento, para que sea efectiva, debe apoyarse ineludiblemente en aquello que piensan con respecto al tema por desarrollar. Por tanto, para el caso de las ideas alternativas sobre 
ecorregión, los estudiantes intentan estructurar una definición a partir de los conceptos ambiente, territorio y región, de los cuales se desprenden los elementos para la construcción de la categoría ecorregión (red de conceptos), como espacio homogéneo, como asociación de conceptos, como sistema de relaciones, como sistema natural, y como espacio de la acción humana y del desarrollo.

De acuerdo con lo anterior, en este trabajo se tuvieron en cuenta los conocimientos e intereses de los estudiantes, con el fin de que el trabajo por desarrollar fuera significativo para su formación profesional.

- En relación con la metodología empleada, cabe resaltar la progresión de las concepciones que presentan los estudiantes frente a la ecorregión. Este proceso evidencia la transición hacia formas más complejas de comprensión por parte de los sujetos, por cuanto se construye un espacio relacional en el que dicho concepto adquiere sentido.

- Consecuentemente, consideramos que el trabajo por talleres y salidas de campo aporta a la conceptualización de las temáticas sobre el sistema agua, bosques, biodiversidad y a la calidad de vida y la sostenibilidad, constituyéndose de esta forma en nodos articuladores que favorecen la construcción individual y colectiva de la categoría ecorregión.

- Educar ambientalmente en el marco de este trabajo no sólo corresponde a desarrollar una labor cognitiva, a interpretar una realidad o a construir un concepto, sino que trasciende al educar desde la vida, desde la realidad de los sujetos, con base en procesos de reflexión que permitan dimensionar su compromiso y responsabilidad, tanto con la sociedad como con la vida, a partir del presente.

- Para fortalecer el sentido que tiene esta categoría en la formación de ciudadanos, vale la pena destacar que, según los principios metodológicos de la investigación-acción, la construcción de la categoría ecorregión posibilita la reflexión permanente y la emergencia de una visión de mundo. Esta categoría intenta complejizar la mirada sobre la dinámica ambiental, al tiempo que su construcción colectiva posibilita una forma de concebir un espacio académico relacional, en el que se pueden identificar los diversos ámbitos de dicha realidad.

- Para la construcción de la categoría ecorregión es fundamental que puedan comprenderse los sistemas ambientales como sistemas dinámicos y complejos cuya organización es un resultado de fluctuaciones a lo largo del tiempo, tal y como se concreta en la ecorregión local de Ubaté. De igual forma, es importante resaltar que el proceso de aprendizaje en los sujetos resulta complejo, puesto que se procura que no sea de forma lineal o acumulativa, sino por medio de interacciones, sinergias que se traduzcan en emergencias, utilizando toda la experiencia cognoscitiva aparte de la valorativa y afectiva que se entretejen en una urdimbre para producir conocimiento.

Vale la pena resaltar como un logro fundamental de la presente investigación, el hecho de que el grupo de 
estudiantes haya construido desde sus comprensiones las conceptualizaciones en torno a la ecorregión, que trascienden significativamente a la definición dada por el Ministerio del Medio Ambiente, Vivienda y Desarrollo Territorial, sintetizando en ella un verdadero aprendizaje significativo.

- El proceso de investigación-acción supone una construcción de conocimientos a partir de la interacción de los sujetos, siendo un enfoque investigativo en el que se propugna lo democrático, lo ético y lo interpretativo; un conocimiento de la realidad que no se piensa como reflejo objetivo de las cosas "externas", sino como una comprensión que lleva en sí el sello de quienes conocen, por lo que la validez de la investigación queda de manifiesto con el diálogo de saberes entre pares cognitivos (profesores, compañeros, miembros de la comunidad), fortaleciendo, de esta manera, una conciencia crítica a través de la retroalimentación y la evaluación permanente del proceso.

- Resulta esencial que los docentes en formación construyan criterios y conocimientos que les posibiliten, por un lado, identificar las complejas problemáticas ambientales, y por otro, la ampliación de redes conceptuales que den cuenta de su visión de mundo. Es en este contexto donde los componentes cultural, sociopolítico y económico exigen un acercamiento para generar oportunidades de cambio, lo cual puede ser un aporte a los propósitos institucionales, dada su proyección en el ámbito social, pues- to que es la escuela -y en este caso la Universidad-, el espacio que favorece la permanente recontextualización de la realidad.

- La conceptualización de la categoría ecorregión se convierte en una posibilidad de construcción de conocimiento y punto de partida para un proceso de profundización teórica, conceptual y metodológica, orientado hacia la comprensión de realidades ambientales, constituyéndose en una propuesta pedagógica y didáctica, para ser abordada desde el escenario escolar. De este modo permite complejizar las miradas acerca del contexto ambiental, sea éste local, regional o nacional.

Así mismo, el abordaje de la ecorregión puede constituirse en una propuesta metodológica de gestión, que involucra a los distintos actores sociales en un proceso de construcción colectiva de conocimiento. Dicha propuesta procura la adquisición de sentido en torno a la complejización del ambiente, a partir de la articulación de los ámbitos de desarrollo sostenible, calidad de vida, conservación y manejo adecuado de los recursos, de tal manera que puedan ser tenidos en cuenta en el ordenamiento territorial, consolidándose como ejes orientadores para el establecimiento de procesos en la búsqueda de desarrollo sostenible, mejoramiento de la calidad de vida, fortalecimiento de acciones de conservación y de manejo adecuado de los recursos, con el fin de ser cristalizados en ecorregiones estratégicas en el país. 


\section{Conceptualización de la categoría ecorregión}

Como se ha mencionado, para el desarrollo de la investigación se parte de la propuesta de ecorregión planteada por el Ministerio de Ambiente, Vivienda y Desarrollo Territorial, la cual se encuentra estructurada a partir de tres criterios: delimitación territorial, jurisdicción de las corporaciones y carácter estratégico, restringiéndose fundamentalmente a una definición. Por tanto, uno de los propósitos del presente artículo consiste en brindar una conceptualización de ecorregión. Los resultados de este proceso de conceptualización han llevado a la construcción de una categoría (sistema de conceptos relacionales), propuesta como un referente teórico-conceptual y metodológico al aportar elementos que permiten, por un lado, su identificación en un contexto ambiental particular, y por otro, una forma de abordar una ecorregión, desde la educación y la gestión ambiental.

Como resultado del proceso de conceptualización desarrollado a partir del presente trabajo, podemos entender la ecorregión como:

Construcción social, referida a una unidad territorial estratégica, concebida como un sistema integral, dinámico y complejo, determinada por el conjunto de interacciones de los subsistemas biofísico (agua, suelo, bosque, biodiversidad, clima, en interrelación) y sociocultural, en un contexto particular, en la cual se circunscriben: el territorio como espacio geográfico, en el que se construye un sistema de valores y creencia; éste dimensiona las identidades culturales, provistas por redes de significados que dan sentidos de pertenencia en el colectivo, creando una proyección que no se circunscribe a lo físico, sino que es esencialmente simbólica. Y la región como un área de la superficie terrestre que se caracteriza por una relativa homogeneidad, producto de procesos biofísicos y socioculturales, así como de las fluctuaciones generadas a partir de las interacciones entre todos sus componentes. En este sentido, la cultura se constituye en espacio propicio para tejer una urdimbre de relaciones que posibilitan la construcción de conocimientos, prácticas, técnicas, valores y procesos de negociación de significados. Estos conocimientos, a su vez, podrían ser la base para nuevas formas de situarse e interactuar éticamente con el colectivo y con el entorno, con el fin de que el colectivo reconozca sus problemáticas y formule posibles soluciones, que se cristalicen mediante el fortalecimiento de una capacidad de gestión, el uso sostenible de los recursos y la satisfacción de las necesidades humanas. Todo esto en la búsqueda de mejorar sustancialmente la calidad de vida de los individuos y contribuir, de esta manera, a la sostenibilidad ambiental, así como a una conservación de la vida a nivel local, regional, nacional y global. $\mathbf{\Delta}$

\section{Bibliografía}

Arnal, Del Rincón y Latorre (1992). Investigación educativa, fundamentos $y$ metodologías. Barcelona: Labor.

Ausubel, D. P. (1978). Psicología educativa. Un punto de vista cognoscitivo. México: Trillas. 
Max Neef (2000). Desarrollo a escala humana. (Editorial). Colombia: Proyecto 20 Editores.

Ministerio del Medio Ambiente (2002). Proyecto Colectivo Ambiental, Colombia.

(2000). Programas para la Implementación del Plan Estratégico. Colombia

Morin, Edgar (1996). Introducción al pensamiento complejo. España: Gedisa.

Moreira, Marco A. (2003). "Lenguaje y aprendizaje significativo". Conferencia de Cierre del IV Encuentro Internacional sobre Aprendizaje significativo. Brasil.

Novo Villaverde, M. (1991). La educación ambiental. España: REI. (2003). La educación ambiental. Bases éticas, conceptuales y metodológicas. Madrid: Universitas, S.A.
Rodríguez. A. (2000). Geografía conceptual. Colombia. Universidad Pedagógica Nacional.

Talero, E. y Umaña. G. (1998). Aportes para la realización del Proyecto de Educación Ambiental. Bogotá: Ediciones Romero.

Torres, Maritza (1996). La dimensión ambiental: un reto para la educación de la nueva sociedad. Bogotá: Ministerio de Educación Nacional.

Umaña, G. (2004). "La pedagogía de terreno". Documento interno de trabajo. Universidad Pedagógica Nacional.

Zubiría, M. et al. (1997). Fundamentos de pedagogía conceptual. Una propuesta curricular para la enseñanza de las Ciencias Sociales para pensar. Bogotá: Plaza \& Janés. 